\title{
The c. $-1639 G>A$ polymorphism of the VKORC1 gene is a major determinant of the response to acenocoumarol in anticoagulated patients
}

\author{
Ramón Montes, ${ }^{1}$ Estefanía Ruiz de \\ Gaona, ${ }^{1}$ Miguel Ángel Martínez- \\ González, ${ }^{2}$ Ignacio Alberca ${ }^{3}$ and José \\ Hermida $^{1}$ \\ ${ }^{1}$ Laboratory of Thrombosis and Haemostasis, \\ Haematology Department and Division of \\ Cardiovascular Sciences, Centre for Applied \\ Medical Research, Clinica Universitaria/School of \\ Medicine, University of Navarra, ${ }^{2}$ Department of \\ Preventive Medicine and Public Health, School of \\ Medicine, University of Navarra, Pamplona, and \\ ${ }^{3}$ Department of Haematology, University Hospital \\ of Salamanca, Salamanca, Spain
}

Received 7 November 2005; accepted for

publication 12 January 2006

Correspondence: José Hermida MD PhD,

Laboratory of Thrombosis and Haemostasis,

Centre for Applied Medical Research, University

of Navarra, C/Pío XII, 55, 3rd floor, 31008

Pamplona, Spain. E-mail: jhermida@unav.es

\begin{abstract}
Summary
Much of the variability in the sensitivity to warfarin in anticoagulated patients is associated with the $c .-1639 G>A$ polymorphism of the vitamin $K$-epoxide reductase (VKORC1) gene. However, its association with the acenocoumarol dose in patients under anticoagulant therapy has not been studied. The c. $-1639 G>A$ genotype of VKORC1 was determined in 113 patients on stable anticoagulation requiring low $(n=42)$, medium $(n=42)$ or high $(n=21)$ acenocoumarol doses. To evaluate the association between acenocoumarol requirements and the $c .-1639 G>A$ variant, multivariate logistic regression models were fitted, adjusting for age, gender, and the c.430C $>T$ and c.1075A $>C$ variants of cytochrome P450 2C9 (CYP2C9). A total of $90.5 \%$ of the patients in the low acenocoumarol dose group carried the $A$ allele of VKORC1:c. $-1639 G>A$. The A allele independently increased the odds of requiring a low acenocoumarol dose [odds ratio (OR) 9.4; 95\% confidence interval (CI) $1.9-46 \cdot 4 ; \quad P=0.006]$, especially when the homozygous form was present (OR 44.2; 95\% CI 5.5-354.6; $P<0 \cdot 001$ ). The $A$ allele was less frequent in the high dose group showing an inverse association with the requirement for high doses (OR 0.04; 95\% CI 0.01-0.22; $P<0.001)$. The $A$ allele of the $c .-1639 G>A$ polymorphism of $V K O R C 1$ is therefore associated with a low-dose requirement for acenocoumarol in patients receiving anticoagulant therapy.
\end{abstract}

Keywords: vitamin K, drugs, anticoagulation.
The coumarin derivative acenocoumarol is widely used to prevent recurrent arterial and venous thrombosis in European and Latin-American countries. The appropriate dose varies greatly and it is difficult to predict on an individual basis. Although this may partly be due to environmental factors, i.e. simultaneous exposure to other drugs that interfere with the metabolism of acenocoumarol, dietary habits or other health conditions, the inter-individual variability is thought to be largely determined by hereditary factors (Meyer, 2000). Among the genetic determinants, the variants $c .430 C>T$ (Arg144Cys) and c.1075A $>C$ (Ile359Leu) of cytochrome P450 2C9 (CYP2C9), involved in the metabolism of warfarin and acenocoumarol, have been shown to influence the anticoagulant dosage (Aithal et al, 1999; Daly \& King, 2005). Progress has recently been made in our knowledge of the vitamin $\mathrm{K}$ cycle: the gene coding for the enzyme responsible for the transformation of vitamin $\mathrm{K}$ 2,3-epoxide to vitamin
K-hydroquinone has been cloned (Li et al, 2004; Rost et al, 2004). Vitamin K-hydroquinone is the co-factor for the carboxylase that adds a molecule of carbon dioxide to glutamic acid, producing $\gamma$-carboxyglutamic acid $(\gamma$-Glu). The $\gamma$-Glu residues are essential for some clotting factors, such as factors II, VII, IX, X and protein C, S and Z, to bind phospholipids and thus be functional. The enzyme, called vitamin K-epoxide reductase (VKORC1), is the target of the coumarin derivatives. Importantly, several genetic variations of the VKORC1 gene have been found to influence sensitivity to warfarin (Rost et al, 2004; D'Andrea et al, 2005; Geisen et al, 2005; Quteineh et al, 2005; Rieder et al, 2005). Twenty-eight polymorphisms have been described in this gene, three main haplotypes covering almost the whole genetic variability of VKORC1. VKORC1*2 haplotype has been found to account for most of the variability in response to oral anticoagulants (Geisen et al, 2005; Rieder et al, 2005; Sconce et al, 2005; Yuan et al, 2005). The 
c. $-1639 G>A$ polymorphism marks the $V K O R C 1^{\star} 2$ haplotype (Geisen et al, 2005), and its association with increased response to acenocoumarol has been shown in a group of healthy subjects (Bodin et al, 2005). However, its influence in patients receiving acenocoumarol treatment have not been studied to date. Our aim was to study the effect of the c. $-1639 G>A$ polymorphism on the acenocoumarol dosage requirements in a group of patients under stable anticoagulation.

\section{Materials and methods}

\section{Participants}

A total of 113 outpatients attending the Hospital Clínico Universitario de Salamanca (Spain) between January and October 2001 who had maintained stable anticoagulation [International normalised ratio (INR) between 2 and 3.2] with acenocoumarol for at least 3 months were included in the study. We considered a patient on stable anticoagulation as one whose acenocoumarol dose requirement had remained constant for at least the previous 3 months, and this dose was the same (variation $<25 \%$ ) as the mean annual dose, and was enough to keep the INR within therapeutic ranges. Two patients with liver disease and two patients with thyroid disease were excluded. Four patients could not be analysed. The final population consisted of 42 consecutive patients requiring a low acenocoumarol dose ( $7 \mathrm{mg} /$ week or less), 42 consecutive patients requiring a medium dose $(>7 \mathrm{mg}$ and $<28 \mathrm{mg}$ per week) and 21 consecutive patients requiring a high dose $(28 \mathrm{mg} /$ week or more). Low, medium and high weekly dose categories were arbitrarily chosen, taking into account the fact that around $80 \%$ of subjects in our population of patients under anticoagulation need a dose within the range defined as the medium dose (Hermida et al, 2002).

Data concerning age, sex, weekly dose required, exposure to other drugs affecting the acenocoumarol metabolism and the genotype of the $c .430 C>T$ and $c .1075 A>C$ variants of CYP2C9 were recorded as described elsewhere (Hermida et al, 2002). All subjects had given informed consent to participate in the study.

\section{VKORC1:c.-1639G > A genotyping}

Genotyping was done by polymerase chain reaction followed by digestion with Msp1 (New England Biolabs, Ipswich, MA, USA) according to described methods with minor modifications (Sconce et al, 2005). The forward and reverse primers were $\quad 5^{\prime}$-GCCAGCAGGAGAGGGAAATA- $3^{\prime}$ and $5^{\prime}$-AGTTTGGACTACAGGTGCCT- $3^{\prime}$ respectively.

\section{Statistical analysis}

In order to evaluate the association between acenocoumarol requirements and the presence of the $A$ allele at the -1639 position in the VKORC1 gene, multivariable logistic regression models were fitted after adjusting for age, gender and the presence of other polymorphisms (CYP2C9:c.430C $>T$ and c. $1075 A>C)$. We fitted two separate models. In both cases, the reference category was the group requiring a medium dose of acenocoumarol. In the first model, we estimated the odds ratio (OR) for requiring a low dose (excluding those with a requirement for a high dose). In the second model, we estimated the OR for requiring a 'high' dose (excluding those with a requirement for a low dose).

In the case of the CYP2C9:c.430C > T and c.1075A >C polymorphisms, heterozygous and homozygous subjects for the allelic variants were merged in the same category due to the low prevalence of homozygous subjects.

A linear regression model was also performed to assess the effect of the $A$ allele of VKORC1:c. $-1639 G>A$ on the weekly dose of acenocoumarol (as a continuous variable) within the low dose group.

Interaction (effect modification) was assessed by introducing product-terms in the logistic regression models and considering any $P$-value $<0.05$ for the likelihood ratio test of this product-term as evidence of a significant interaction.

In order to assess the proportion of carriers of both VKORC1:c. $-1639 G>A$ and c.1075A $>C$ polymorphisms requiring a low acenocoumarol dose, we used exact methods [exact binomial confidence intervals (CI) and exact Fisher test]. Statistical analyses were performed with STATA v9 software (StataCorp LP, College Station, TX, USA).

\section{Results}

Effect of VKORC1:c.-1639G > A on acenocoumarol dosage requirement

The distribution of the $G$ and $A$ alleles of $c .-1639 G>A$ polymorphism is shown in Table I. The allelic distribution was different across the various acenocoumarol dosage groups. Remarkably, in the group of 42 patients who maintained stable anticoagulation with a low dose of acenocoumarol, 38 patients

Table I. Frequency of the different genotypes for the $c .-1639 G>A$ polymorphism of the VKORC1 gene in three groups of patients with different acenocoumarol requirements.

\begin{tabular}{lccc}
\hline \multicolumn{4}{c}{ Acenocoumarol dose requirement } \\
\cline { 2 - 4 } & $\begin{array}{l}\text { Low dose } \\
(n=42)\end{array}$ & $\begin{array}{l}\text { Medium dose } \\
(n=42)\end{array}$ & $\begin{array}{l}\text { High dose } \\
(n=21)\end{array}$ \\
\hline$G G$ & $4(9 \cdot 5 \%)$ & $10(23 \cdot 8 \%)$ & $15(71 \cdot 4 \%)$ \\
$G A$ & $12(28 \cdot 6 \%)$ & $24(57 \cdot 1 \%)$ & $6(28 \cdot 6 \%)$ \\
$A A$ & $26(61 \cdot 9 \%)$ & $8(19 \cdot 1 \%)$ & $0(0 \%)$ \\
\hline
\end{tabular}

The number of carriers of each VKORC1:c. $-1639 G>A$ genotype, and the percentage (parentheses) of each genotype for each acenocoumarol dose are represented.

$P$ for trend, $<0 \cdot 001$. 
(90.5\%) carried the $A$ allele. However, the $A$ allele was less frequent in the group of 21 patients requiring a high acenocoumarol dose: among these patients only 6 (28.6\%) carried the $A$ allele in the heterozygous form and none of them carried the $A A$ genotype.

The mean age and the $c .430 C>T$ and $c .1075 A>C$ variants of CYP2C9 in the three groups of patients have been reported previously (Hermida et al, 2002). We showed that both age and the polymorphisms of $C Y P 2 C 9$, especially the $c .1075 A>C$ variant, influenced the acenocoumarol dose requirements, while gender and other drugs did not (Hermida et al, 2002). We fitted logistic regression models to assess the independent contribution of VKORC1:c. $-1639 G>A$ to the inter-individual variability of the pharmacological response to acenocoumarol, taking also into account the effect of polymorphisms of CYP2C9, of age and sex. The results of this analysis are shown in Table II. The presence of the $A$ allele increased the odds of requiring a low acenocoumarol dose. This effect was notably higher when the $A$ allele was present in the homozygous form: OR 44.2 (95\% CI 5.5-354.6; $P<0 \cdot 001$ ). Moreover, an inverse correlation was seen between the mean weekly dose of acenocoumarol required and bearing at least one VKORC1:c. $-1639 A$ allele ( $\beta$ coefficient, $-0.35 ; P=0.043$ ) within the low dose group. Accordingly, not only $\operatorname{did}$ the $A$ allele influence the odds of requiring a low acenocoumarol dose, but it also exerted an independent effect against requiring a high acenocoumarol dose.

The sensitivity and specificity of carrying the VKORC1:c. $-1639 A$ allele for low dose requirements were $90 \cdot 5 \%$ (95\% CI $77 \cdot 4-97 \cdot 3)$ and $40 \%(95 \%$ CI $27 \cdot 6-52 \cdot 8)$ respectively.

\section{Effect of age and the variants of CYP2C9}

The effect of age and the $c .430 C>T$ and $c .1075 A>C$ variants of CYP2C9 on the acenocoumarol requirement in this

Table II. Association of the VKORC1:c. $-1639 G>A$ polymorphism, the $c .430 C>T$ and $c .1075 A>C$ variants of $C Y P 2 C 9$ and age with the likelihood of requiring a low (A) or a high (B) dose of acenocoumarol.

\begin{tabular}{|c|c|c|c|c|c|}
\hline & $\begin{array}{l}\text { Crude } \\
\text { OR }\end{array}$ & $95 \%$ CI & $\begin{array}{l}\text { Adjusted } \\
\mathrm{OR}^{*}\end{array}$ & $95 \% \mathrm{CI}$ & $P$-value \\
\hline \multicolumn{6}{|l|}{ A: low dose } \\
\hline VKORC1:c. $-1639 A$ & $2 \cdot 96$ & $0 \cdot 85-10 \cdot 37$ & $9 \cdot 38$ & $1 \cdot 90-46 \cdot 43$ & $0 \cdot 006$ \\
\hline CYP2C9 variants & $2 \cdot 28$ & $0 \cdot 95-5 \cdot 50$ & $3 \cdot 79$ & $1 \cdot 24-11 \cdot 60$ & $0 \cdot 020$ \\
\hline Age (years) & $1 \cdot 12$ & $1 \cdot 05-1 \cdot 20$ & $1 \cdot 16$ & $1 \cdot 07-1 \cdot 25$ & $<0 \cdot 001$ \\
\hline \multicolumn{6}{|l|}{ B: high dose } \\
\hline VKORC1:c. $-1639 A$ & $0 \cdot 12$ & $0 \cdot 04-0 \cdot 41$ & $0 \cdot 04$ & $0 \cdot 01-0 \cdot 22$ & $<0 \cdot 001$ \\
\hline CYP2C9 variants & $0 \cdot 21$ & $0 \cdot 04-1 \cdot 07$ & $0 \cdot 06$ & $0 \cdot 01-0 \cdot 62$ & $0 \cdot 017$ \\
\hline Age (years) & $0 \cdot 94$ & $0.89-0.99$ & $0 \cdot 90$ & $0.84-0.97$ & $0 \cdot 005$ \\
\hline
\end{tabular}

OR, odds ratio; CI, confidence interval.

${ }^{\star}$ Logistic regression models adjusted for gender and for the other variables shown in the table. The group of patients taking the medium dose of acenocoumarol was considered the reference category in both models. population has been analysed and the results are available elsewhere (Hermida et al, 2002). However, at the time that work was carried out, the polymorphisms of the VKORC1 gene had not yet been identified. For this reason, we updated the estimates for the independent association between both age and the variants of CYP2C9 and the odds of requiring a low acenocoumarol dose after also adjusting for the VKORC1:c. $-1639 G>A$ polymorphism in the multivariate analysis. As can be observed in Table II, age was still an independent factor influencing the acenocoumarol dose after adjusting for the c. $-1639 G>A$ polymorphism: every additional year of age was associated with $16 \%$ higher odds of needing a lower acenocoumarol dose. Bearing the c.430C $>T$ or c.1075A > C CYP2C9 variants also remained independently associated with a higher odds of requiring a low acenocoumarol dose.

\section{Combined effect of the A allele of VKORC1:c.-1639G > A} and the CYP2C9 variants

Remarkably, within the group requiring low acenocoumarol doses, all patients $(n=42)$ were carriers of at least one of the three polymorphisms analysed. In contrast, among the 21 patients who required a high acenocoumarol dose, 13 of them $(61.9 \%)$ were free of the three polymorphisms. In the medium dose group, 36 of 42 subjects carried at least one of the three polymorphisms. All patients who were simultaneously carriers of both the VKORC1:c. $-1639 G>A$ allele and the $C Y$ P2C9:c.1075A $>C$ polymorphism $(n=10)$ required a low acenocoumarol dose, whereas 11 of 20 patients (55\%) carrying the $A$ allele of VKORC and CYP2C9:c.430C $>T$ belonged to the low acenocoumarol dose group. When an exact method (binomial CI) was used to assess the proportion of carriers of both VKORC1:c.-1639A and CYP2C9:c.1075A > C polymorphisms requiring a low acenocoumarol dose, the 95\% CI estimated that $69-100 \%$ of patients that carried both mutations would require a low acenocoumarol dose $(P<0.001$, Fisher exact test).

\section{Discussion}

The recent identification of the VKORC1 gene (Li et al, 2004; Rost et al, 2004), involved in the vitamin $\mathrm{K}$ cycle, has prompted the search for new polymorphisms that can contribute to explaining the high interindividual variability in the dose requirements for oral anticoagulants. Much of this variability has been found to be due to the VKORC1 genotype. Notably, $37 \%$ of the total variability in response to acenocoumarol in a group of healthy subjects was reported to be attributable to VKORC1 (Bodin et al, 2005). Although several haplotypes have been identified in the VKORC1 gene, those including the c. $-1639 G>A$ polymorphism have been associated with variability in the sensitivity to warfarin or acenocoumarol (Bodin et al, 2005; D'Andrea et al, 2005; Geisen et al, 2005; Rieder et al, 2005). Our findings support the importance of the role 
played by the genotype $c .-1639 G>A$ in the sensitivity to acenocoumarol. The present study found that carrying the $A$ allele notably increased the odds of requiring a low acenocoumarol dose to reach an INR within the therapeutic range. More than $90 \%$ of the patients who required a low dose of acenocoumarol to maintain a therapeutic INR carried the $A$ allele, while this allelic variant was only present in $<30 \%$ of the patients who needed a high dose. This allele was present in $76 \%$ of the patients in the medium dose group. Our findings support the notion that the association of VKORC1:c. $-1639 G>A$ with the sensitivity to acenocoumarol is larger than that observed with the CYP2C9 polymorphisms, as the probability of requiring a low acenocoumarol dose associated with carrying the A allele was almost three times higher than the probability associated with the CYP2C9 variants.

Although the characteristics of the present study did not allow us to determine how the variability differs for each polymorphism, from our data it can be inferred that genotyping VKORC1:c. $-1639 G>A$ and CYP2C9: c.1075A $>C$ can help to identify a high percentage of the patients who can safely commence and maintain their anticoagulation on a low dose of acenocoumarol without risk of experiencing a bleeding episode. The 10 patients who simultaneously carried the $A$ allele of VKORC1:c. $-1639 A>G$ and CYP2C9:c.1075A $>C$ required a low dose of acenocoumarol for stable anticoagulation. Accordingly, among the 42 consecutive patients who entered the study because they required a low dose of acenocoumarol, 40 of them carried at least one of the two polymorphisms.

Our findings could be relevant when applied to clinical practice: as it has been suggested that the hyper-responsiveness to oral anticoagulant is linked to high risk of bleeding at the beginning of treatment, patients to be treated with acenocoumarol and bearing the $c .-1639 G>A$ polymorphism of VKORC1 should start treatment with a reduced initial 'loading' dose in order to avoid over-anticoagulation and subsequent bleeding risk, particularly if they also carry the c.1075A >C variant of CYP2C9. An algorithm to calculate the daily dose of warfarin taking into account the VKORC1 genotype has recently been proposed (Sconce et al, 2005). The present study could not build an algorithm because our population was not randomly selected.

The higher sensitivity to coumarin derivatives in the presence of the $A$ allele of the VKORC1:c. $-1639 G>A$ polymorphism is probably due to a lower amount of VKORC1 in these patients, which would lead to a decrease in the availability of vitamin K-hydroquinone: recently, an association between low hepatic VKORC1 mRNA levels and haplotypes of the VKORC1 gene carrying the $-1639 A$ allele has been shown, such patients being particularly sensitive to warfarin (Rieder et al, 2005).

This study has limitations: the sample size, although large enough to draw conclusions, was not sufficient to avoid wide CI; on the other hand, the patients were not randomly selected but chosen according to predefined groups (i.e. low, medium and high acenocoumarol dose requirements). Hence, our results cannot be directly applied to the general population of patients receiving anticoagulants. Nonetheless, the strength of the association between the VKORC1:c. $-1639 G>A$ polymorphism and the acenocoumarol requirements undoubtedly underlines the importance of this genetic variant as a major determinant of the sensitivity to acenocoumarol in patients under anticoagulant treatment.

In conclusion, for the first time the association between the c. $-1639 G>A$ polymorphism of the VKORC1 gene and the acenocoumarol requirement for stable long-term anticoagulation has been studied. The $A$ allele increases the likelihood of requiring a low dose of acenocoumarol, especially when the c.1075A > C variant of the CYP2C9 gene is also present. Genotyping for both mutations should make it possible to identify the vast majority of patients that are likely to require a low dosage of acenocoumarol.

\section{Acknowledgements}

This project was funded through the Unión Temporal de Empresas (UTE) project Centro de Investigación Médica Aplicada (CIMA). We also received funding from Instituto de Salud Carlos III (Red temática de investigación RECAVA, C03/ 01) and from Ministerio de Ciencia y Tecnología (SAF200308706), Spain.

\section{References}

Aithal, G.P., Day, C.P., Kesteven, P.J. \& Daly, A.K. (1999) Association of polymorphisms in the cytochrome P450 CYP2C9 with warfarin dose requirement and risk of bleeding complications. Lancet, 353, 717-719.

Bodin, L., Verstuyft, C., Tregouet, D.A., Robert, A., Dubert, L., FunckBrentano, C., Jaillon, P., Beaune, P., Laurent-Puig, P., Becquemont, L. \& Loriot, M.A. (2005) Cytochrome P450 2C9 (CYP2C9) and vitamin $\mathrm{K}$ epoxide reductase (VKORC1) genotypes as determinants of acenocoumarol sensitivity. Blood, 106, 135-140.

D’Andrea, G., D'Ambrosio, R.L., Di Perna, P., Chetta, M., Santacroce, R., Brancaccio, V., Grandone, E. \& Margaglione, M. (2005) A polymorphism in the VKORC1 gene is associated with an interindividual variability in the dose-anticoagulant effect of warfarin. Blood, 105, 645-649.

Daly, A.K. \& King, B.P. (2005) Pharmacogenetics of oral anticoagulants. Pharmacogenetics, 13, 247-252.

Geisen, C., Watzka, M., Sittinger, K., Steffens, M., Daugela, L., Seifried, E., Muller, C.R., Wienker, T.F. \& Oldenburg, J. (2005) VKORC1 haplotypes and their impact on the inter individual and inter-ethnical variability of oral anticoagulation. Thrombosis and Haemostasis, 94, 773-779.

Hermida, J., Zarza, J., Alberca, I., Montes, R., López, M.L., Molina, E. \& Rocha, E. (2002) Differential effects of $2 \mathrm{C} 9^{*} 3$ and $2 \mathrm{C} 9^{\star} 2$ variants of cytochrome P-450 CYP2C9 on sensitivity to acenocoumarol. Blood, 99, 4237-4239.

Li, T., Chang, C.Y., Jin, D.Y., Lin, P.J., Khvorova, A. \& Stafford, D.W. (2004) Identification of the gene for vitamin K epoxide reductase. Nature, 427, 541-544. 
Meyer, U.A. (2000) Pharmacogenetics and adverse drug reactions. Lancet, 356, 1667-1671.

Quteineh, L., Verstuyft, C., Descot, C., Dubert, L., Robert, A., Jaillon, P. \& Becquemont, L. (2005) Vitamin K epoxide reductase (VKORC1) genetic polymorphism is associated to oral anticoagulant overdose. Thrombosis and Haemostasis, 94, 690-691.

Rieder, M.J., Reiner, A.P., Gage, B.F., Nickerson, D.A., Eby, C.S., McLeod, H.L., Blough, D.K., Thummel, K.E., Veenstra, D.L. \& Rettie, A.E. (2005) Effect of VKORC1 haplotypes on transcriptional regulation and warfarin dose. New England Journal of Medicine, 352, 2285-2293.

Rost, S., Fregin, A., Ivaskevicius, V., Conzelmann, E., Hortnagel, K., Pelz, H.J., Lappegard, K., Seifried, E., Scharrer, I., Tuddenham, E.G.,
Muller, C.R., Strom, T.M. \& Oldenburg, J. (2004) Mutations in VKORC1 cause warfarin resistance and multiple coagulation factor deficiency type 2. Nature, 427, 537-541.

Sconce, E.A., Khan, T.I., Wynne, H.A., Avery, P., Monkhouse, L., King, B.P., Wood, P., Kesteven, P., Daly, A.K. \& Kamali, F. (2005) The impact of CYP2C9 and VKORC1 genetic polymorphism and patient characteristics upon warfarin dose requirements: proposal for a new dosing regimen. Blood, 106, 2329-2333.

Yuan, H.Y., Chen, J.J., Lee, M.T., Wung, J.C., Chen, Y.F., Charng, M.J., Lu, M.J., Hung, C.R., Wei, C.Y., Chen, C.H., Wu, J.Y. \& Chen, Y.T. (2005) A novel functional VKORC1 promoter polymorphism is associated with inter-individual and inter-ethnic differences in warfarin sensitivity. Human Molecular Genetics, 14, 1745-1751. 